\title{
Short communication: Detection and monitoring of metritis in dairy cows using an automated grooming device
}

\author{
R. Mandel, ${ }^{*} \dagger^{1}$ C. J. Nicol, $\ddagger$ H. R. Whay, $\ddagger$ and E. Klement* \\ *Koret School of Veterinary Medicine, Robert H. Smith Faculty of Agriculture, Food and Environment, The Hebrew University, Rehovot 76100, \\ Israel \\ †Department of Environmental Systems Science. Institute of Agricultural Sciences, ETH Zürich, Zurich 8092, Switzerland \\ ¥School of Veterinary Science. University of Bristol, BS40 5DU, United Kingdom
}

\section{ABSTRACT}

Metritis, a prevalent disease on dairy farms, is negatively associated with reproduction, milk production, and the welfare of cows. The objective of this study was to evaluate the efficacy of monitoring low-resilience activities (i.e., behaviors that typically decrease when energy resources are limited or when the cost involved in the activity increases; e.g., brush usage) in the early detection of metritis. Data on daily brush usage (i.e., proportion of cows using the brush and the duration of usage) were collected from 28 metritic and 60 control cows 28 d postpartum using an automated monitoring system developed for the purpose of this study. During the first week following partum (before clinical diagnosis), we found no differences in brush usage between sick and control cows. However, 8 to $21 \mathrm{~d}$ postpartum (the week of clinical diagnosis and the first week of medical treatment), a lower proportion of metritic cows used the brush compared with control cows (0.49 compared with 0.64 , respectively, at brushes installed away from the feed bunk). In addition, the daily duration of brush usage was $50 \%$ lower among cows diagnosed with metritis compared with control cows 8 to 28 d postpartum (44 s/d compared with $88 \mathrm{~s} / \mathrm{d}$, respectively). The results of this study suggest that on-farm monitoring of low-resilience behaviors, combined with existing systems that monitor core behaviors (e.g., activity and rumination), may serve as an improved method for detecting events that compromise the welfare of animals. The slow recovery of low-resilience behaviors following medical treatment (wk 4) might serve as a particularly useful indicator of progress of recovery from disease.

Key words: automated brush, cattle welfare, lowresilience behavior, sickness behavior

Received October 25, 2016.

Accepted March 13, 2017.

${ }^{1}$ Corresponding author: roi.mandel@mail.huji.ac.il

\section{Short Communication}

On the modern dairy farm, technology is commonly used to assist farmers with feeding, milking, detecting estrus behavior, and moving cows through automated individual recognition gates. A more recent trend is the use of technology to monitor the health state of individual cows by monitoring changes in physiological (e.g., milk yield, milk pH, BW, body temperature) and behavioral (e.g., rumination and activity meters) parameters associated with prevalent diseases such as clinical mastitis, lameness, and metabolic problems (reviewed in Rutten et al., 2013). Existing systems focus on measuring changes in the most basic and essential behaviors of cows (also referred to as core behaviors), such as those that are associated with food consumption (rumination tags) and basic features of movement (number of steps per time unit and lying duration as measured with activity loggers). By focusing on changes in core behaviors (e.g., daily duration of rumination), a low rate of false alarms can be achieved, thus reducing the chances of farmers neglecting the system's warning signs. However, because these types of behaviors are essential for the short-term survival of the animal, they are expected to decrease only at a relatively later stage of disease (Littin et al., 2008), to facilitate a fever response ("sickness behavior"; Hart, 1988), or when a sufficient degree of pain or lethargy is induced. The cost of such an approach is thus the low level of sensitivity during the early stages of disease, when physical signs of illness are poorly pronounced. An additional challenge that arises with the use of core behaviors as a measure of health condition is their rapid recovery following medical treatment. Once an animal is treated for an illness and the levels of core behaviors recover back to baseline, the animal is assumed to be fully recovered and therefore fit to be integrated back into the production line.

An alternative approach that could potentially improve detection of illness and provide more information on the recovery phase is to combine on-farm measures 
of core behaviors with measures of low-resilience behaviors (also referred to as luxury activities-i.e., behaviors that typically decrease when energy resources are limited or when the cost involved in the activity increases; McFarland, 1999; see also Dawkins, 1990). Low-resilience behaviors are expected to decrease earlier than core activities and could therefore enable early detection of diseases (Littin et al., 2008; Weary et al., 2009). By monitoring change in low-resilience behaviors (e.g., the use of climbing resources such as a rope and ladder) in female R6/1 transgenic Huntington's disease mice, researchers were able to detect signs that precede clinical signs of disease (Littin et al., 2008). In addition, low resilience behaviors would be expected to have a longer recovery phase, compared with core behaviors, which can recover rapidly following medical treatment (e.g., mastitis; Sepúlveda-Varas et al., 2014). The aim of the current cohort study was to determine the association between brush usage and metritis in the first $28 \mathrm{~d}$ following partum, before and after medical treatment. Our hypotheses were that cows diagnosed with metritis would use the brush less compared with healthy cows and that the use of distant brushes (i.e., brushes installed away from the feed bunk) would be more indicative of morbidity than the use of brushes that were installed adjacent to the feed bunk (i.e., the difference in brush usage between the sick group and the control group would be higher; Mandel et al., 2013).

The study was carried out at a commercial dairy farm in the northern Negev of Israel from May 2015 to February 2016 (9 mo). Brushing activity was collected from 240 Holstein dairy cows. Among these, 106 cows calved during that period. Cows were kept in 3 groups according to their lactation status (first lactation, second lactation, and third lactation or higher). All groups were kept in loose-housing cowsheds bedded with dried manure. First, second, and third or higher lactation groups (70-80 cows/group) were housed in $90.0 \times 16.6 \mathrm{~m}$ cowsheds. Cows were fed a TMR twice a day at 0800 and 1600 . Water was available ad libitum from 6 water troughs $(18 \mathrm{~cm}$ of trough/cow) in each cowshed. Cows were milked 3 times a day at 0400 to 0600,1100 to 1300 , and 1900 to 2100 . A routine veterinary examination was carried out at 7 to $14 \mathrm{~d}$ postpartum for all cows that calved during the data collection period ( $\mathrm{n}=106$ cows) with the aim of detecting signs of metritis. After the perineal area was thoroughly cleaned and disinfected, each cow was comprehensively examined through intravaginal and transrectal palpation by the farm's veterinary surgeon assigned by Hachaklait Veterinary Services Ltd. (Caesarea, Israel). Cases of retained fetal membranes were defined as the presence of placental tissues $24 \mathrm{~h}$ or more after calving as observed by a trained farm employee or the attending veterinarian. Ketosis was diagnosed by placing a drop of urine obtained with a disposable plastic catheter on a reagent strip (Ketostix, Ames, England) and comparing the color of the reaction after $15 \mathrm{~s}$ with a standardized color chart. Cows with urine acetoacetate concentration $\geq 15 \mathrm{mg} / \mathrm{dL}$ were recorded as ketotic (Goshen and Shpigel, 2006). Cows diagnosed with retained fetal membranes or other postpartum diseases (e.g., ketosis) and health risk conditions (e.g., lameness) were excluded from the statistical analysis. In the present study, we did not differentiate between puerperal metritis and clinical metritis. Clinical diagnosis of metritis was based on characteristics of vaginal discharge (obtained by manual examination of the vagina) and on cervical and uterine examination by palpation of the rectum. Affected cows had a flaccid, nonretractable uterus that was located in the abdomen; a cervical diameter of $>75 \mathrm{~mm}$; and a watery or purulent, fetid vaginal discharge (Goshen and Shpigel, 2006). All cows diagnosed with metritis were treated with $5 \mathrm{~g}$ of chlortetracycline i.u. twice per week for $2 \mathrm{wk}$ (Goshen and Shpigel, 2006). Dates of disease diagnosis and medical treatment (provided by the farm's veterinarian) were collected from the Israeli management information system for the national dairy and beef herds. Clinical examination revealed signs of metritis in 37 out of 106 cows that calved during the course of the experiment. Nine cows diagnosed with metritis were additionally diagnosed with ketosis, retained fetal membranes, or lameness and were therefore excluded from the analysis. Among the 28 metritic cows included in the analyses, 13 cows were in their first lactation and 15 cows were in their second lactation or higher. The control group comprised 60 (out of 69) cows that calved during the course of the experiment and were found to be negative for metritis, lameness, or other postpartum diseases. Among the control cows, 20 cows were in their first lactation and 40 cows were in their second lactation or higher.

Six months prior to the beginning of the experiment, 6 rotating brushes (swinging cow brush SCB, DeLaval International $\mathrm{AB}$, Tumba, Sweden) were installed in the dairy farm. Two brushes were installed in each group. To improve the detection of stress and morbidity (Mandel et al., 2013), 1 brush was installed $3 \mathrm{~m}$ from the food bunk (referred to as the near brush) and the other was installed $16 \mathrm{~m}$ from the food bunk, on the opposite side of the yard (referred to as the distant brush).

Data on daily brush usage (in seconds) were collected automatically using a brush monitoring system tailored for this purpose. The system was based on the technology of a commercial rumination and activity monitoring system (SCR, Natanya, Israel) that was reprogrammed 
and modified in a way that allowed us to monitor the time and duration (temporal resolution $=1 \mathrm{~Hz}$ ) of use, the identity of cows standing under or in close proximity to the brush (radius of $1 \mathrm{~m}$ ), and whether the brush was rotating. Validation of the brush monitoring system was carried out in 2 sessions of direct observations ( $4 \mathrm{~h}$ each) conducted by a single observer (from 0800 to 1200 and from 1300 to $1700 \mathrm{~h}$ ). A total of 270 time samples (capturing 18 brushing events conducted by 13 cows) were recorded using a 1-0 time sampling method with a 5-s interval (Altmann, 1974). The system was able to identify $75 \%$ (203/270 time samples) of brushing events (i.e., when a cow was standing next to the brush and the brush rotating function was turned on). No false positives were recorded because the tags worn on the necks of the cows could transmit an identification signal only after being activated via infrared light. Infrared light beams were limited to $1 \mathrm{~m}$ from the brush and were activated only when motion was detected under the brush and when the brush rotating function was turned on.

To reduce false registration of brush usage (i.e., when a cow was crossing next to the brush with no clear intention to use it), the data were retained for analysis only if a cow was present in a radius of $1 \mathrm{~m}$ from the brush (based on the range of the infrared beam) for at least $10 \mathrm{~s}$ and if the rotating function of the brush turned on at least once during this time period. Tensecond intervals were chosen to minimize incidences of false registration of brush usage as mentioned previously and because brush rotation function was set by the manufacturer to continue for $10 \mathrm{~s}$ after the last activation. During the calibration of the system, we noticed that when a cow was using the brush continuously for at least $10 \mathrm{~s}$, the system, which was originally set to sample the surroundings every second $(1 \mathrm{~Hz})$, would at times miss detecting the presence of the cow in 1 or 2 continuous samples. We therefore allowed a maximum gap of $3 \mathrm{~s}$ between 2 continuous readings to minimize the chance of losing any brushing events due to the technical limitations of the system. The data produced by the system $\log$ files were filtered, processed, and saved to a database (Microsoft SQL Server, 2012 version; Microsoft Inc., Redmond, WA).

To adequately reflect dependencies in the experimental design (nesting, repeated measurements), linear and generalized linear mixed-effects models were used to evaluate the outcome variables. Statistical analysis was performed in R 3.0.2 software (lmer and glmer functions, lme4 library; http://www.R-project.org). To identify the most indicative measuring unit, brush usage was analyzed as daily duration of brush usage $(\mathrm{s} / \mathrm{d})$ and as daily occurrence (binary; $0=$ no use; $1=$ used at least once). These 2 variables were entered as outcome variables in different models, once for both brush locations (close or distant to food bunk) and for each brush location separately. For each model, the explanatory factors were days from calving (continuous) and health status (2 levels: metritis or control). Daily brush usage on the day of partum was not included in the analysis because of the effect of calving time on the availability of the brush. Lactation was not included in the model because of its collinearity with group (pen). Random effects included cow identity nested within group. Date of observation was added as a crossed-random effect (random effect that applied independently from the nested effect) because the disease period of some cows overlapped with others and to control for differences in temperature and humidity between days. To assess the effect of metritis before and after medical treatment, we used different models to assess the overall effect of metritis (d 1-28) and the effect per week [i.e., the week preceding clinical diagnosis (wk 1), the week of diagnosis (wk 2), and the first (wk 3) and second (wk 4) weeks of medical treatment]. For each model, we assessed the statistical significance of the fixed effects by comparing the model with and without the factor included using likelihood ratio tests. The likelihood ratio test statistics follow a $\chi^{2}$ distribution with degrees of freedom equal to the difference in the number of parameters. Nonsignificant terms were removed using a standard model simplification procedure (i.e., stepwise backward elimination). The significance level was set at $\alpha=0.05$. Tendency was defined as $0.05<P<0.1$. The residuals were checked graphically for normal distribution and homoscedasticity. To satisfy assumptions, we used a $\log$ transformation for daily duration of brush usage. Results are presented as mean \pm standard deviation.

Throughout the observation period (28 d postpartum), the proportion of cows using a brush was significantly lower among cows diagnosed with metritis $(0.75 \pm 0.43)$ compared with the control group $(0.85 \pm$ $\left.0.36, \chi^{2}{ }_{1}=5.21, P=0.023\right)$. Daily occurrence of brush usage at brushes installed away from the feed bunk was significantly lower among cows diagnosed with metritis $(0.49 \pm 0.50)$ compared with the control group $(0.62$ $\left.\pm 0.48, \chi_{1}{ }_{1}=5.74, P=0.017\right)$. However, daily occurrence of brush usage at brushes installed adjacent to the feed bunk did not differ between cows diagnosed with metritis $(0.59 \pm 0.49)$ and the control group (0.66 $\left.\pm 0.47, \chi_{1}^{2}=2.14, P=0.14\right)$. When splitting the data by week (i.e., the week preceding clinical diagnosis, the week of diagnosis, and the first and second weeks of medical treatment), we found that a smaller proportion of metritic cows used the brushes installed away from the feed bunk compared with control cows during wk 2 and 3 , and a similar tendency was found during wk $4(P$ $=0.0505$; Table 1$)$. However, the groups did not differ 
Table 1. Use of brushes installed in 2 locations (away from and next to food) in sick (metritis) and healthy cows (mean \pm SD) ${ }^{1}$

\begin{tabular}{|c|c|c|c|c|c|c|c|c|}
\hline Item & \multicolumn{4}{|c|}{ Next to food (low cost) } & \multicolumn{4}{|c|}{ Away from food (high cost) } \\
\hline \multicolumn{9}{|l|}{$\overline{\mathrm{Wk}} 1$} \\
\hline Duration $^{2}$ & $83 \pm 142$ & $85 \pm 127$ & 0.31 & 0.58 & $64 \pm 117$ & $125 \pm 276$ & 0.28 & 0.59 \\
\hline \multicolumn{9}{|l|}{ Wk 2} \\
\hline Proportion & 0.60 & 0.69 & 2.36 & 0.12 & 0.48 & 0.66 & 7.45 & $0.006^{* *}$ \\
\hline Proportion & 0.63 & 0.62 & 0.02 & 0.89 & 0.48 & 0.64 & 6.02 & $0.014^{*}$ \\
\hline Duration & $86 \pm 154$ & $75 \pm 140$ & 0.07 & 0.80 & $37 \pm 68$ & $83 \pm 139$ & 7.88 & $0.005^{* *}$ \\
\hline \multicolumn{9}{|l|}{ Wk 4} \\
\hline Proportion & 0.56 & 0.61 & 1.22 & 0.27 & 0.50 & 0.62 & 3.83 & 0.0505 \\
\hline Duration & $50 \pm 91$ & $70 \pm 151$ & 0.28 & 0.60 & $44 \pm 78$ & $75 \pm 131$ & 4.26 & $0.039^{*}$ \\
\hline
\end{tabular}

${ }^{1} \chi^{2}$ distribution with $1 \mathrm{df}$.

${ }^{2}$ Duration of brush usage is represented as seconds per day.

${ }^{*} P<0.05,{ }^{* *} P<0.01$.

during wk 1 (before clinical diagnosis of disease). For brushes installed adjacent to the feed bunk, we found no significant differences between metritic and control cows (Table 1).

Throughout the observation period (28 d postpartum), daily duration of brush usage was significantly lower among cows diagnosed with metritis (118.1 $\pm 171.5)$ compared with the control group (169.6 \pm $\left.235.9 \mathrm{~s} / \mathrm{d}, \chi^{2}{ }_{1}=4.93, P=0.026\right)$. Daily duration of use of brushes installed away from the feed bunk was significantly lower among cows diagnosed with metritis (48.6 \pm 92.0) compared with the control group (96.6 $\left.\pm 195.2 \mathrm{~s} / \mathrm{d}, \chi_{1}^{2}=5.36, P=0.021\right)$. Daily duration of use of brushes installed adjacent to the feed bunk did not differ between cows diagnosed with metritis $(72.3 \pm 132.5 \mathrm{~s} / \mathrm{d})$ and the control group $(74.4 \pm 134.2$ $\left.\mathrm{s} / \mathrm{d}, \chi_{1}^{2}=0.74, P=0.39\right)$. When splitting the data by week, daily duration of brush use was significantly lower for metritic cows compared with control cows in brushes installed away from the feed bunk during wk 2,3 , and 4 . However, the groups did not differ during wk 1 (before clinical diagnosis of disease; Table 1). For brushes installed adjacent to the feed bunk, we found no significant differences between metritic and control cows (Table 1).

The objective of this study was to evaluate the efficacy of monitoring low-resilience activities in the early detection of metritis. Reduced levels of brush usage were found in metritic cows compared with control cows 8 to $28 \mathrm{~d}$ postpartum during the week of diagnosis (wk 2) and in the first (wk 3) and second (wk 4) weeks of medical treatment. Differences in brush usage between metritic and control cows were documented mainly in brushes that were installed distantly from the feed bunk and not in those installed adjacent to the feed bunk. Prioritization of behaviors during a state of a disease was envisaged by Hart (1988), who claimed that mammals and birds facilitate the role of fever in the battle against viral and bacterial infections by reorganizing their behavior ("sickness behavior"; Hart, 1988). According to Hart (1988), the reduced activity expressed during the state of a disease (e.g., lethargy, sleepiness, and depression) is part of a strategy aimed to save energy (and minimize heat loss), allowing animals to cope with the energetic costs of fever (estimated to increase metabolism by up to 50\%; Hart, 1988). In our study, prioritization of behavior was documented during the early stages of the disease even in the absence of fever (all metritic cows but 1 had no signs of elevated body temperature).

In our previous study (Mandel et al., 2013), reduced brush usage was more indicative of stress (i.e., heat load) on days when food was located away from the feed bunk compared with days when food was located next to the brush. A similar pattern of results was found in the present study, with the use of distant brushes being more indicative of disease than the use of close brushes. This finding corresponds to the notion that behaviors with a primary function of promoting the survival of the individual (e.g., feeding) will precede behaviors that promote other aspects of fitness (e.g., brush usage in our case; McFarland, 1999). This finding, together with previous findings (Mandel et al., 2013), suggests that there is a trade-off between brush location and the sensitivity of this tool for detecting stress and morbidity.

According to the theory of behavioral resilience and demand curves (Dawkins, 1990; McFarland, 1999), compared with core behaviors, low-resilience behaviors are expected to decrease earlier when energy resources are limited or when the cost involved in the activity increases. According to this logic, once an animal is 


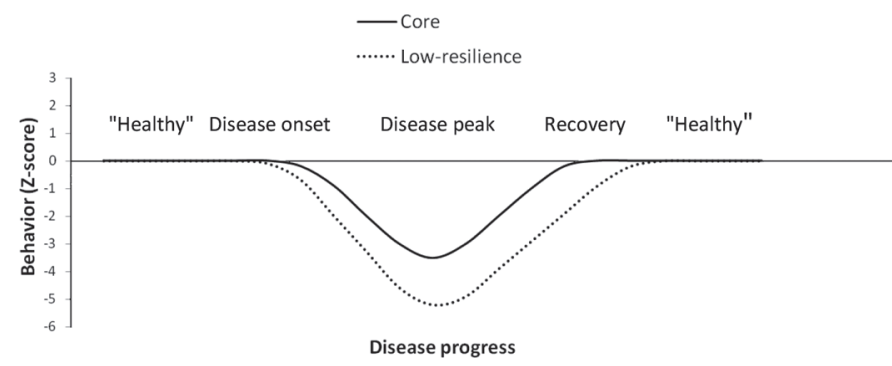

Figure 1. Hypothetical model of the changes in core and lowresilience behaviors as a function of disease progression. Changes in low-resilience behaviors (e.g., brush usage) are expected to (1) precede changes in core behaviors (e.g., activity and rumination), (2) decrease to a greater magnitude compared with core behaviors, and (3) have a delayed return to baseline levels following medical treatment compared with core behaviors.

treated for illness and energy resources are beginning to recover, core behaviors are expected to have a shorter recovery phase compared with low-resilience behaviors. In our study, after 2 wk of medical treatment, brush usage levels of cows treated for metritis did not recovery fully and were still lower than those of the control cows, demonstrating the potential use of low-resilience behaviors for assessing a more complete recovery from disease and injury (Figure 1). We encourage the further assessment of this tool, specifically when combined with measures of core behaviors for improving the sensitivity of existing automated health monitoring systems in detecting the onset of disease and for assessing recovery.

\section{ACKNOWLEDGMENTS}

We thank the Hoffmann leadership program (Hebrew University, Jerusalem, Israel) and the Universities Fed- eration for Animal Welfare, Support of Animal Welfare in Israel (UFAW-SAWI) fund for funding R. M. and this research. We also thank Elodie F. Briefer (Institute of Agricultural Sciences, ETH Zürich, Zurich, Switzerland) and 2 anonymous reviewers for commenting on a previous version of this article.

\section{REFERENCES}

Altmann, J. 1974. Observational study of behavior: Sampling methods. Behaviour 49:227-267.

Dawkins, M. S. 1990. From an animal's point of view: Motivation, fitness, and animal welfare. Behav. Brain Sci. 13:1-9.

Goshen, T., and N. Y. Shpigel. 2006. Evaluation of intrauterine antibiotic treatment of clinical metritis and retained fetal membranes in dairy cows. Theriogenology 66:2210-2218.

Hart, B. L. 1988. Biological basis of the behavior of sick animals. Neurosci. Biobehav. Rev. 12:123-137.

Littin, K., A. Acevedo, W. Browne, J. Edgar, M. Mendl, D. Owen, C. Sherwin, H. Würbel, and C. Nicol. 2008. Towards humane end points: Behavioural changes precede clinical signs of disease in a Huntington's disease model. Proc. R. Soc. Lond. (Biol.) 275:18651874

Mandel, R., H. R. Whay, C. J. Nicol, and E. Klement. 2013. The effect of food location, heat load, and intrusive medical procedures on brushing activity in dairy cows. J. Dairy Sci. 96:6506-6513.

McFarland, D. 1999. Animal Behaviour. 3rd ed. Addison Wesley Longman, Reading, UK.

Rutten, C. J., A. G. J. Velthuis, W. Steeneveld, and H. Hogeveen. 2013. Invited review: Sensors to support health management on dairy farms. J. Dairy Sci. 96:1928-1952. https://doi.org/10.3168/ jds.2012-6107.

Sepúlveda-Varas, P., K. L. Proudfoot, D. M. Weary, and M. A. von Keyserlingk. 2014. Changes in behaviour of dairy cows with clinical mastitis. Appl. Anim. Behav. Sci. 175:8-13.

Weary, D. M., J. M. Huzzey, and M. A. G. Von Keyserlingk. 2009. Board-invited review: Using behavior to predict and identify ill health in animals. J. Anim. Sci. 87:770-777. 